\title{
The effect of intrinsic muscular nonlinearities on the energetics of locomotion in a computational model of an anguilliform swimmer
}

Christina Hamlet ${ }^{1, *}$, Lisa J. Fauci ${ }^{1}$ Eric D. Tytell ${ }^{2}$,

1 Department of Mathematics, Tulane University, New Orleans, LA, USA

2 Department of Biology, Tufts University, Medford, MA, USA

* Corresponding author email: chamlet@tulane.edu (CLH)

\begin{abstract}
Animals move through their environments using muscles to produce force. When an animal's nervous system activates a muscle, the muscle produces different amounts of force depending on its length, its shortening velocity, and its time history of force production. These muscle forces interact with forces from passive tissue properties and forces from the external environment. Using an integrative computational model that couples an elastic, actuated model of an anguilliform, lamprey-like swimmer with a surrounding Navier-Stokes fluid, we study the effects of this coupling between the muscle force and the body motion. Swimmers with different forms of this coupling can achieve similar motions, but use different amounts of energy. The velocity dependence is the most important property of the ones we considered for reducing energy costs and helping to stabilize oscillations. These effects are strongly influenced by how rapidly the muscle deactivates; if force decays too slowly, muscles on opposite sides of the body end up fighting each other, increasing energy cost. Work-dependent deactivation, an effect that causes a muscle to deactivate more rapidly if it has recently produced mechanical work, works together with the velocity dependence to reduce the energy cost of swimming.
\end{abstract}

(C) 2015. This manuscript version is made available under the Elsevier user license http://www.elsevier.com/open-access/userlicense/1.0/ 


\section{Introduction}

Locomotion is a basic behavior of animals, yet even the simplest motions require the coordination of many systems, including the nervous system, musculoskeletal system, sensory systems, and even the external environment 1 3]. Feedback is critical to maintaining coordination 4]. Animals have different forms of feedback that are important for movement. The most commonly recognized type of feedback is when an animal senses its environment and changes its motor pattern. Another form of feedback is purely mechanical: animals' bodies are flexible and deform in response to external forces before the nervous system has time to respond [2]. Our focus is on a second form of mechanical feedback where muscle force development depends on muscle length, rate of contraction, and time history [5]. Together, these forms mechanical feedback are sometimes called "preflexes", because they can occur before a neural reflex 6]. These classes of responses help animals respond rapidly, effectively, and efficiently to their environment.

Previously, we developed a computational model of the flexible body of a swimming lamprey 7 . This model simulated the coupling between internal passive mechanical forces, muscular forces, and the external fluid environment. We showed swimming motion is strongly dependent on the coupling between the body and the environment. For example, there is an optimal body stiffness for maximum steady swimming speed 7]. This model only included mechanical feedback but not nonlinearities that underlie muscle force development.

These nonlinearities include the relationships between muscle force, its length and its shortening velocity [5. If a muscle is clamped at a constant length and stimulated (an isometric contraction), its active force depends on the length, with lower force at short or long lengths and a maximum at some optimal length in between. This effect is called the force-length relationship ( $\lambda$, Figure 1 A). If the muscle is then allowed to shorten (a concentric contraction), the force will also depend on the shortening speed, decreasing as the shortening speed gets higher. If the muscle is lengthened by some external force, even as it produces force to resist the lengthening (an eccentric contraction), the active force increases above the isometric value. Together, these effects are called the force-velocity relationship of a Hill-type model $(\alpha$, Figure 1B $)$ ). We use the standard assumption that muscle force can be modeled by a product of the length dependence, the velocity dependence, and an activation level that captures the amount of calcium that is bound to myosin filaments in the muscle 8 11.

Recently, researchers have described two other effects that influence muscle force. If the muscle 

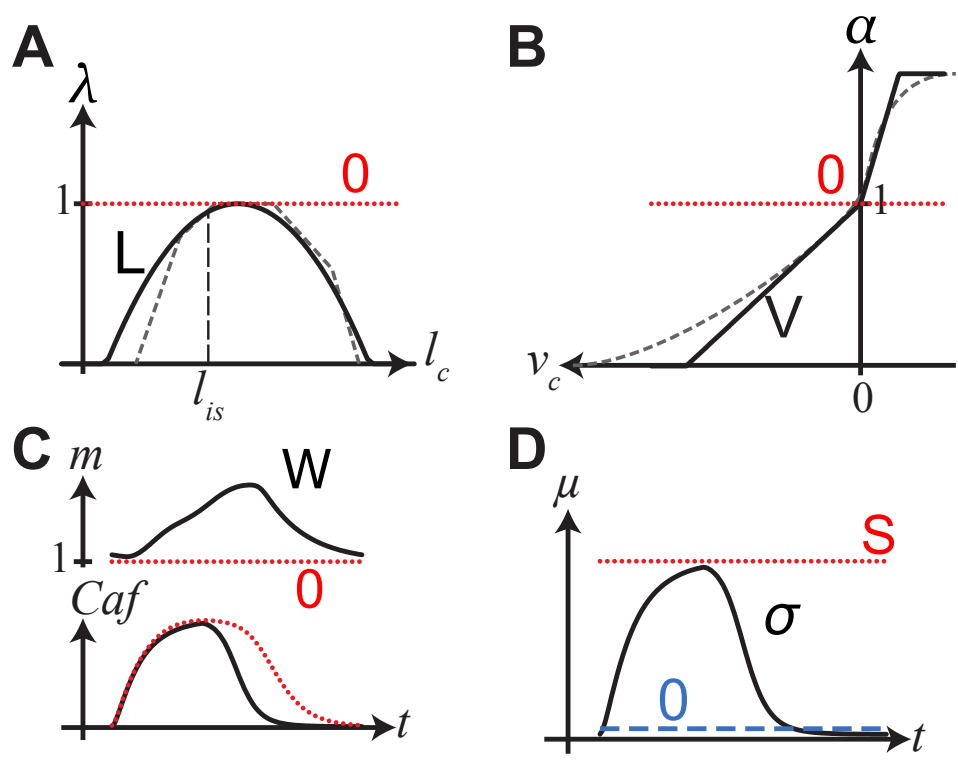

Figure 1. Schematic of four nonlinearities. In each panel, the black curve shows the nonlinear effect and the red curve shows the value when the effect was disabled. In the top two panels, the dashed gray curves depict typical experimental measurements. Each curve also has the letter code used to identify different simulations. A. Force-length relationship $\lambda\left(l_{c}\right)$. B. Force-velocity relationship $\alpha\left(v_{c}\right)$. C. Work-dependent deactivation. The top panel shows $m(t)$, the integrated muscle work and the bottom panel shows how it speeds up decrease in muscle activation Caf. D. Calcium-dependent stiffness. The black curve shows the variable stiffness $\mu(\mathrm{Caf})$ and the red and blue curve show the high and low constant values.

produces mechanical work while active, it deactivates more rapidly when the activation stops [12, an effect called "work-dependent deactivation" (WDD; Figure 1C). Following Williams [9], we model WDD by integrating the muscle power $(\mathrm{m})$ and increasing the deactivation rate according to the amount of work the muscle recently produced. "Passive" muscle stiffness also changes when the muscle is active, due to calcium-dependent effects on the titin molecule that links myosin filaments 13 15] (Figure1D). We incorporate a series elastic element with a stiffness $\mu$ that depends on the bound calcium concentration Caf (after Williams 9]).

In static conditions, non-linear dependencies contribute to stabilizing both muscle length and force. For example, the standard operating length of many muscles, including lamprey muscle, is in the rising limb of the force-length curve (Figure 1A) 5, 10, 16. If a perturbation lengthens the muscle a small amount, it produces more force because it is longer and because the force increases when the muscle is forcibly lengthened, both of which help it to shorten [6]. More broadly, several studies have found that intrinsic muscle properties can stabilize standing or postural responses 17, 18. 
The role of intrinsic muscular properties in cyclic motions is not well understood. Haeufle et al. $[19$ studied a mathematical model of hopping, driven by a Hill-type muscle model. They found that the forcevelocity relationship stabilized the hopping height to perturbations, but reduced the maximum achieved hopping height.

These intrinsic muscular properties vary across species and different muscle fiber types, and can be altered by degree of activation, exercise or aging. For example, Woledge 20 found that tortoise skeletal muscle has a much more steeply curved force-velocity relationship than frog muscle, which he suggested contributed to a more efficient conversion of energy into mechanical work 20]. In some fishes, the shape of the force-velocity relationship can change at different temperatures 21], although for others, the shape remains the same over a broad temperature range 22]. Different levels of activation can also change the shape of the curves: for instance, the force-length curve of frog muscle becomes narrower at a low stimulation intensities 23]. Finally, exercise and aging can alter the properties. The optimal length can shift after eccentric exercise [24], and both elderly people and stroke victims have altered forcelength and force-velocity properties (e.g., $25 \mid 26]$ ). The calcium-dependent effects of titin in changing the muscle stiffness has been identified fairly recently, and so there is relatively little information on how it varies across species or muscle types. Lampreys have titin, but its structure may be different from the mammalian form [27].

We developed our model to investigate the function of the different forms of intrinsic muscular properties. Rather than attempting to simulate differences, described above, we instead added or removed the properties, to better understand their overall effects, both individually and in combination with each other.

\section{Models}

We developed a two-dimensional model of an anguilliform swimmer that includes a fully nonlinear muscle model (after [9]) coupled to a flexible body immersed in a Navier-Stokes fluid (extending [7]). Below, we describe (1) the muscle model, then (2) the immersed boundary framework for fluid-structure interactions, and (3) the quantification of energetics of the model swimmer. Figure 2 shows the overall structure of the model, with references to the sections below.

The muscle segments produce force $P_{c}$ which is applied to the fluid-structure model that also in- 


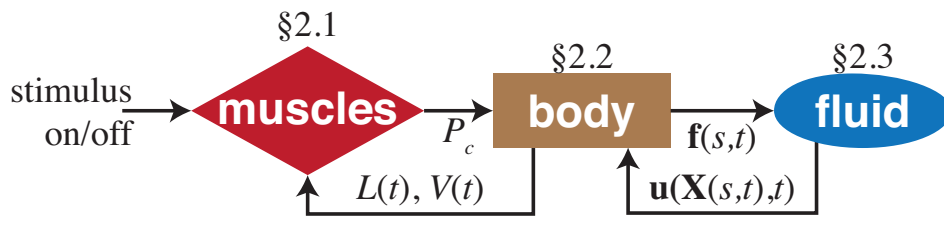

Figure 2. A schematic of the coupled muscle, body, and fluid elements in the full model. Section numbers are given for each block and arrows are labeled with the value connecting the different blocks in the model.

corporates passive mechanics to produce a total force $\mathbf{f}(s, t)$, where $s$ parameterizes the body whose configuration at a given time $t$ is $\mathbf{X}(\mathrm{s}, \mathrm{t})$. The Navier-Stokes model allows the calculation of the fluid velocity at the body $\mathbf{u}(\mathbf{X}(s, t), t)$, and thus describes how body segments change length. The segment lengths $L(t)$ and their velocities $V(t)=\dot{L}(t)$ are fed back as parameters for the muscle model.

\subsection{Muscle Model}

The muscles produce force according to a dynamical system modified from [9] that couples calcium activation to a Hill-type muscle model. A Hill-type model assumes that muscle force can be approximated as the product of a length-dependent term, a velocity-dependent term, and an activation term, and that a muscle segment is comprised of a contractile element in series with a passive elastic element, in parallel with another passive elastic element. Since its development, the Hill model has been modified and used as a kinematic model for muscle force development; for example see 9 . Here we choose a two-element model of a muscle segment: a contractile element in series with an elastic element as shown in Figure 3 We incorporate four nonlinearities (Figure 1): the classical force-length and force-velocity relationships 8, 9], a work-dependent nonlinearity [12], and a calcium-dependent change in passive stiffness 14,15 .

The force developed by the muscle depends on the length and velocity of the contractile element and the amount of calcium bound to the fibers:

$$
P_{c}=\lambda\left(l_{c}\right) \alpha\left(v_{c}\right) C a f
$$

Here $l_{c}$ is the length of the contractile element normalized by the segment rest length $L_{0}$ and $v_{c}$ is the velocity of the contractile element divided by $L_{0}$ (with this choice of scaling, $l_{c}$ is dimensionless and $v_{c}$ has dimensions of $\left.s^{-1}\right)$. Length dependence is given by $\lambda\left(l_{c}\right)$ and velocity dependence is given by $\alpha\left(v_{c}\right)$, 


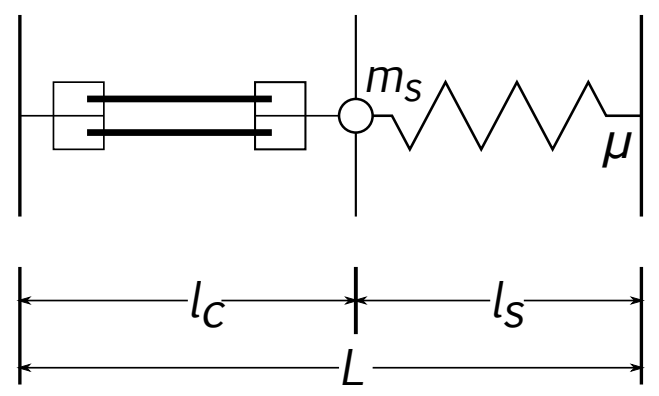

Figure 3. A schematic of the elements of a single muscle segment (sarcomere) in the model. The contractile element $(\mathrm{CE})$, on the left with length $l_{c}$, contracts when the activation wave provides calcium to the segment. The series elastic element, on the right with length $l_{s}$ consisting of a Hookean spring and a dashpot, stores and releases energy from work done by the CE.

and Caf is the level of activation based on the amount of calcium bound to the muscle fibers. Note that $\lambda\left(l_{c}\right), \alpha\left(v_{c}\right)$, and $C a f$ are dimensionless, and the contractile force $P_{c}$ has also been made nondimensional by scaling it by the maximum tetanic force $P_{0}$. Equation 1 is used to drive a spring-mass-damper system

$$
m_{s} \ddot{l}_{s}+d_{s} \dot{l}_{s}+\mu(\text { Caf }) l_{s}=P_{c}
$$

where $l_{s}$ is the nondimensional length of the series elastic element (scaled by $L_{0}$ ) (Figure 3), $m_{s}$ is the scaled mass of the sarcomere (following [28) with dimensions $\mathrm{s}^{2}, d_{s}$ is a damping coefficient with dimensions s, and $\mu(C a f)$ is the nondimensional spring constant of the elastic element in Figure 3 . We note that the damping term $\left(d_{s} \neq 0\right)$ has been added as numerical device to dampen out small oscillations in $l_{s}$ that would otherwise develop an instability in the explicit integration of Equation 2. However, in all simulations presented here, the value of $d_{s}$ chosen is small enough that the system remains underdamped and matches biological data in [9]. The spring constant $\mu(\mathrm{Caf})$ depends on the level of calcium binding:

$$
\mu(C a f)=\mu_{0}+\mu_{1} C a f(t) .
$$

We follow Williams 9 in using the following functional forms for force and length dependencies: 


$$
\begin{gathered}
\lambda^{*}\left(l_{c}\right)=1+\lambda_{2}\left(l_{c}-l_{c 0}\right)^{2} \\
\alpha^{*}\left(v_{c}\right)=1+ \begin{cases}\alpha_{m} v_{c} & v_{c} \leq 0 \\
\alpha_{p} v_{c} & v_{c}>0\end{cases}
\end{gathered}
$$

such that:

$$
\lambda\left(l_{c}\right)= \begin{cases}\lambda_{\min } & \text { if } \lambda^{*}\left(l_{c}\right)<\lambda_{\min } \\ \lambda^{*}\left(l_{c}\right) & \text { if } \lambda_{\min } \leq \lambda^{*}\left(l_{c}\right) \leq \lambda_{\max } \\ \lambda_{\max } & \text { if } \lambda^{*}\left(l_{c}\right)>\lambda_{\max }\end{cases}
$$

and

$$
\alpha\left(v_{c}\right)= \begin{cases}\alpha_{\min } & \text { if } \alpha^{*}\left(v_{c}\right)<\alpha_{\min } \\ \alpha^{*}\left(v_{c}\right) & \text { if } \alpha_{\min } \leq \alpha^{*}\left(v_{c}\right) \leq \alpha_{\max } \\ \alpha_{\max } & \text { if } \alpha^{*}\left(v_{c}\right)>\alpha_{\max }\end{cases}
$$

The parameters $l_{c 0}, \lambda_{2}$, and $\alpha_{m}, \alpha_{p}$, and the cutoff values $\lambda_{\min }, \lambda_{\max }, \alpha_{\min }, \alpha_{\max }$ were derived from experiments and reported in 9, 10, where force development on single myomeres from freshly killed lampreys were measured and modeled.

The total length of a given segment

$$
L(t)=l_{c}(t)+l_{s}(t)
$$

evolves according to the body and fluid interaction, described below.

We model the calcium dynamics in a single segment using the mass action model of [10]. When muscle is activated, calcium flows from the sarcoplasmic reticulum (SR) and binds to troponin molecules 
which change conformation to allow myosin to bind to actin and produce force. After the activation, the sarcoplasmic reticulum actively takes up the calcium [5,10]. Calcium release, binding, and reuptake is described by

$$
\begin{aligned}
\frac{d C a}{d t}= & \left(k_{4} C a f-k_{3} C a\right)(1-C a f) \\
& + \begin{cases}k_{1}(C-C a-C a f) & \text { stimulus on } \\
k_{2}(C a(C-S-C a-C a f)) & \text { stimulus off }\end{cases} \\
\frac{d C a f}{d t}=-\left(k_{4} C a f-k_{3} C a\right)(1-C a f) &
\end{aligned}
$$

where $C a$ and $C a f$ are the free calcium and bound calcium on filament sites scaled by the total number of calcium binding sites on the thick filaments, respectively, and $k_{1}-k_{4}$ are the rate constants of the reactions. $\mathrm{S}$ is the ratio of calcium binding sites on the SR to the total number of calcium binding sites on the thick filaments, and $\mathrm{C}$ is the ratio of the total amount of calcium in the system to the number of calcium binding sites on the thick filaments. Both $S$ and $C$ are taken to be constants. To ensure there is enough calcium to bind to all the muscle sites and there are enough sites in SR to sequester all of the calcium during deactivation, the values $\mathrm{C}=2$ and $\mathrm{S}=6$ were chosen, following 10 . Note that, although the total amount of calcium in the system is conserved, the sum of $\mathrm{Ca}$ and $\mathrm{Caf}$ is not constant because there is also calcium that is bound to sites on the SR.

Following [9], to account for work dependent deactivation in the calcium binding dynamics in Eq.10, the values of $k_{3}$ and $k_{4}$ change depending on how much work the muscle has performed, where $m$ is a dimensionless indicator: 


$$
\begin{aligned}
k_{3} & =k_{30} / \sqrt{m} \\
k_{4} & =k_{40} \sqrt{m} \\
\frac{d m}{d t} & = \begin{cases}-k_{m 1} P_{c} v_{c} & v_{c}<0 \\
-k_{m 2}(m-1) & v_{c} \geq 0\end{cases}
\end{aligned}
$$

Note that $m$ increases during muscle shortening, and when the muscle is not shortening, $m$ decays to its initial value of one (1).

The ordinary differential equations for the length of the series elastic element $l_{s}$ (Equation 2), the free and fiber-bound calcium $C a, C a f$ (Equations 9, 10), and the indicator of work performed $m$ (Equation 13. evolve independently on each muscle segment using the initial conditions $l_{s}(0)=0, i_{s}(0)=0, C a(0)=$ $0, \operatorname{Caf}(0)=0$ and $m(0)=1$. The dynamics are driven by an imposed wave of neural activation passed along the series of muscle segments. This activation wave determines whether the stimulus is 'on' or 'off' in the evolution of free calcium (Equation 9).

Model fitting: Williams et al. 10 developed a model of force development and fitted it to experimental data collected by measuring the force developed on a sinusoidally forced lamprey muscle segment. The segment was excised from the lamprey body, sinusoidally stretched and shortened with a frequency $1 \mathrm{~Hz}$. During the sinusoidal motion the muscle was stimulated for a duration of $0.36 \mathrm{~s}$ starting at ten different phases, $\phi$. The experimental results for the 10 phases identified by $0 \leq \phi \leq 1$ are shown in Figure 4 (data provided courtesy of T.L. Williams). To validate our model, we forced a single simulated muscle segment at the same amplitude, frequency and phases as the experiments in Williams et al. 9. The parameters $k_{1}, k_{2}, k_{30}, k_{40}, k_{m 1}, k_{m 2}, m_{s}$, and $d_{s}$ were fitted to the experimental data using a nonlinear least squares minimization routine in Matlab (lsqnonlin). Although this minimization method is iterative, the final values of parameters computed were not sensitive to initial guesses. Parameter values and their dimensions are given in Table 1. A comparison of the model and data are shown in Figure 4. 
Table 1. Parameters used in the muscle model.

\begin{tabular}{lll}
\hline Parameter & Value & Dimensions \\
\hline$\alpha_{m}$ & 0.80 & $\mathrm{~s}$ \\
$\alpha_{\max }$ & 1.8 & \\
$\alpha_{\min }$ & 0 & \\
$\alpha_{p}$ & 2.90 & $\mathrm{~s}$ \\
$\lambda_{2}$ & -20 & \\
$\lambda_{\max }$ & 1 & \\
$\lambda_{\min }$ & 0 & \\
$\mu_{0}$ & 1 & \\
$\mu_{1}$ & 23 & \\
$\boldsymbol{d}_{\boldsymbol{s}}$ & $\mathbf{0 . 2 8 0 2}$ & $\mathrm{s}$ \\
$\boldsymbol{k}_{\mathbf{1}}$ & $\mathbf{6 . 7 2 8 1}$ & $\mathrm{s}^{-1}$ \\
$\boldsymbol{k}_{\mathbf{2}}$ & $\mathbf{2 3 . 2 7 9 4}$ & $\mathrm{s}^{-1}$ \\
$\boldsymbol{k}_{\mathbf{3 0}}$ & $\mathbf{5 1 . 3 5 3 7}$ & $\mathrm{s}^{-1}$ \\
$\boldsymbol{k}_{\mathbf{4 0}}$ & $\mathbf{1 9 . 3 8 0 1}$ & $\mathrm{s}^{-1}$ \\
$\boldsymbol{k}_{\boldsymbol{m} \mathbf{1}}$ & $\mathbf{1 7 . 5 8 0 4}$ & \\
$\boldsymbol{k}_{\boldsymbol{m} \mathbf{2}}$ & $\mathbf{6 . 0 1 5 6}$ & $\mathrm{s}^{-1}$ \\
$l_{c 0}$ & 0.91 & \\
$\boldsymbol{m}_{\boldsymbol{s}}$ & $\mathbf{0 . 0 5 4 2}$ & $\mathrm{s}^{2}$ \\
$C$ & 2 & \\
$S$ & 6 & \\
$P_{0}$ & 60.86 & $\mathrm{mN} \cdot \mathrm{mm}^{-2}$ \\
\hline
\end{tabular}

Bold parameters were fitted to data provided courtesy of T. L. Williams; other parameters are from Williams 9 and McMillen et al. 29.

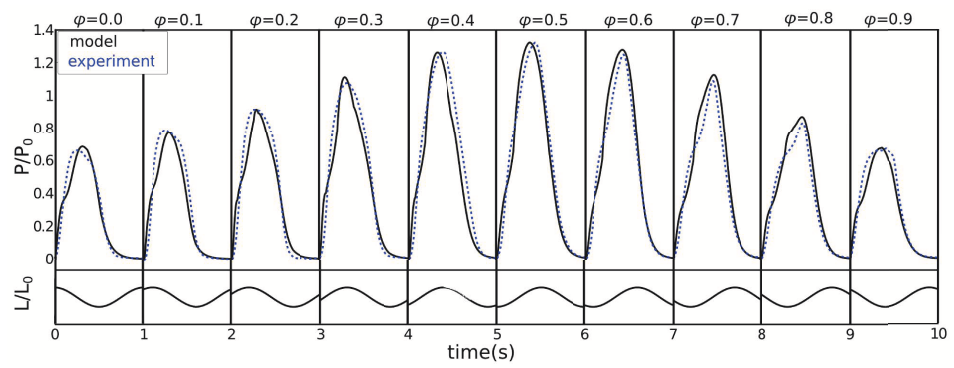

Figure 4. Comparison of the single muscle model response to experimental data. The simulations (black curve) were performed using parameters from Table 1 with a sinusoidally forced segment. The experimental data (dotted blue curve) were recorded from a similarly forced isolated segment. The length of segment for the experimental and numerical data is shown below the force plots. The experimental values $P$ and $L$ have been scaled by the corresponding $P_{0}$ and $L_{0}$ to match $P_{c}$ and $L(t)$ in the numerical simulations. Muscles were stimulated for $0.36 \mathrm{~s}$, starting at the phase shown in each panel. 


\subsection{Body construction and muscle activation}

Using an immersed boundary framework [30], we model a lamprey body as a neutrally-buoyant, elastic, actuated structure comprised of three segmented filaments (see Figure 5). The filament along the midline is stiff, and is connected by springs to the more flexible lateral filaments. Each filament generates forces due to passive elastic properties. The internal links are treated as Hookean springs. However, the links on the lateral edges exert elastic forces because they resist extension, but, like collagen, do not resist compression. In all simulations presented below, the passive stiffnesses of these links are not varied, and the overall Young's modulus of the elastic body is $E=0.76 \mathrm{MPa}$. Note the lateral links also coincide with the muscle segments, and support the contractile forces that have been described above. As in Tytell et al. [7], the model lamprey is constructed using 320 links along each lateral side, and 640 links along the midline, in a conformation that approximates an idealized 2D lamprey of length $12.56 \mathrm{~cm}$.

The activation stimulus for a single muscle segment is imposed in this model, and is incorporated into the mass action equation for calcium (Equation 9). Except for a passive head region at the first twelve percent of the lamprey body, an activation wave along the body is imposed as a step function with a duty cycle of 0.36 , a temporal period of $1 \mathrm{~s}$, such that one third of each lateral side is active at any given time. Moreover, there is a period of silence between the activation waves on the same side and on differing sides 7 .

\section{$2.3 \quad$ Fluid-structure interaction}

Each node on the three filaments comprising the lamprey body supports forces due to the passive, elastic links and the nodes on the lateral filaments also support the contractile forces of the muscle segments that are connected to that node. The muscle model is incorporated into the body structure as a sequence of individually contracting segments on either side of the body. Because both the passive elastic links and the contracting muscle segments contribute equal and opposite forces to the endpoint nodes, the model lamprey is a free-swimmer that generates zero total force and torque at each instant. These forces

$\mathbf{F}(\mathbf{X}, t)$ at the body position $\mathbf{X}$ at time $t$ are coupled to the surrounding incompressible fluid using the immersed boundary formulation: 


$$
\begin{array}{r}
\rho\left[\frac{\partial \mathbf{u}(\mathbf{x}, t)}{\partial t}+\mathbf{u}(\mathbf{x}, t) \cdot \nabla \mathbf{u}(\mathbf{x}, t)\right]=-\nabla p(\mathbf{x}, t)+\mu \nabla^{2} \mathbf{u}(\mathbf{x}, t)+\mathbf{f}(\mathbf{x}, t) \\
\nabla \cdot \mathbf{u}(\mathbf{x}, t)=0 \\
\mathbf{f}(\mathbf{x}, t)=\int_{\Gamma} \mathbf{F}(\mathbf{X}(s, t), t) \delta(\mathbf{x}-\mathbf{X}(s, t)) d s \\
\frac{\partial \mathbf{X}}{\partial t}=\mathbf{u}(\mathbf{X}(s, t), t)=\int_{\Omega} \mathbf{u}(\mathbf{x}, t) \delta(\mathbf{x}-\mathbf{X}(s, t)) d \mathbf{x}
\end{array}
$$

Equations 14 and 15 are the Navier-Stokes equations for a viscous, incompressible Newtonian fluid, where $\mathbf{u}$ is the fluid velocity field, $p$ is the pressure, $\rho=1 \mathrm{~g} \cdot \mathrm{cm}^{-3}$ is the density, $\mu=1 \mathrm{mPa} \cdot \mathrm{s}$ is the fluid viscosity, and $\delta$ is the two-dimensional Dirac delta function. Equation 16 transmits forces from the neutrally-buoyant lamprey body $\Gamma$ to the surrounding fluid domain $\Omega$, and Equation 17 states the points of the immersed lamprey move at the fluid velocity evaluated at those points (no-slip condition).

These equations are discretized using an Eulerian grid for fluid quantities, and a Lagrangian description of the immersed boundary. A grid-dependent, regularized version of the Dirac delta function allows communication between the background finite-difference grid and the nodes of the lamprey body [30,31]. In order to achieve high resolution near the lamprey that allows us to capture the boundary layers generated at physiological Reynolds numbers, we use the parallel, adaptive-mesh implementation of the immersed boundary method developed by Griffith [31. In addition to using a finer mesh around the lamprey body, regions in the fluid domain that exceed a threshold level of vorticity are also tagged for refinement.

We choose a rectangular fluid domain $\Omega=X \times Y$ that is 7.5 body lengths long and 3.0 body lengths high. The coarsest discretization is a 32 cell grid in the $x$-direction over the entire domain, and allowing 5 levels of refinement results in the finest level of effectively a 512 cell grid in the $x$-direction, realized only in the local area of refinement. No-stress boundary conditions are imposed on the rectangular boundary. Each simulation was allowed to run for 10 seconds of simulated time, the duration of 10 activation waves along the body. In each simulation the fluid velocity field is initialized at rest and the lamprey is initialized in the horizontal rest configuration shown in Figure 5 


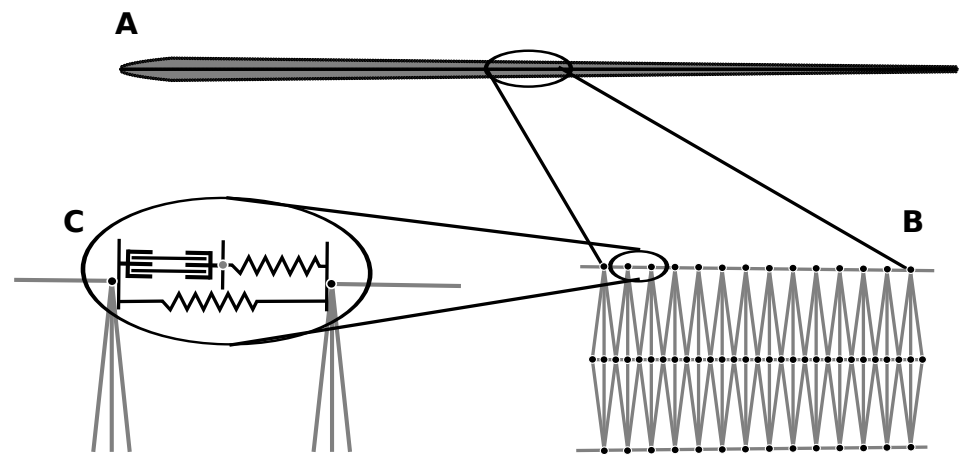

Figure 5. A schematic of the lamprey body. (A) Shape of the entire body. (B) Immersed boundary points (black dots), are connected by springs (thin lines). The diagonal springs model passive body resistance. (C) A close-up of one side showing the position of the muscle model. A spring resisting extension but not compression is added parallel to the muscle model simulating the effects of collagen in the skin.

\section{$2.4 \quad$ Energy calculations}

To quantify the energetics of the model swimmer, we compute the work done by each muscle segment at each time step, separating positive and negative work:

$$
\begin{aligned}
W_{i} & =\left(-P_{c} V\right) \\
\left(W_{+}\right)_{i} & =\left\{\begin{array}{cc}
W_{i} & W_{i}>0 \\
0 & W_{i} \leq 0
\end{array}\right. \\
\left(W_{-}\right)_{i} & = \begin{cases}W_{i} & W_{i}<0 \\
0 & W_{i} \geq 0\end{cases}
\end{aligned}
$$

Here $i$ designates the $i^{t h}$ segment, $P_{c}$ is the contractile force at that segment given by Equation 1, and $V=\dot{L}(t)$ is the velocity of shortening of the entire segment. The muscle work rate, $P$, is calculated using weighted contributions from the positive work $\left(W_{+}\right)_{i}$ and the negative work $\left(W_{-}\right)_{i}$ during an integral number $(k)$ of cycle periods of duration $T$ after the motion has reached steady state (after $n$ cycles):

$$
P=\int_{n T}^{(n+k) T}\left(\sum_{i}\left(\left|W_{+}\right|_{i}+\omega\left|W_{-}\right|_{i}\right)\right) d t
$$


Because the metabolic cost of negative work has been shown to be considerably less than the metabolic cost of positive work, we approximated the metabolic cost of producing the mechanical work by summing the positive work with a fraction of the negative work. We varied $\omega$ from 0 to 1 , and found the proportion of negative work added does not change the relative costs among the simulations. In selecting a representative value for $\omega$ for the results we chose $\omega=0.2$ as a reasonable value based on results in Ruina et al. [32].

The cost of transport $(C o T)$ and the power coefficient $\left(C_{P, \text { mus }}\right)$ are defined as:

$$
\begin{aligned}
C o T & =\bar{P} /\left(U f m_{b}\right) \\
P_{0} & =\frac{1}{2} \rho c U^{3} \\
C_{P, \text { mus }} & =\bar{P} / P_{0}
\end{aligned}
$$

where $\bar{P}$ is the muscle work rate averaged over steady cycles, $f=1 \mathrm{~Hz}$ is the cycle frequency, $m_{b}$ is body mass, $P_{0}$ is the work rate to overcome drag at the steady swimming speed $U$ and the body perimeter $c$. Because we assume the immersed lamprey is neutrally buoyant, the body density $\rho$ is taken to be the same as the fluid density.

\subsection{Kinematic parameters}

In this model, the kinematics of the swimming lamprey are not prescribed, but emerge from the coupling of the elastic, actuated body with the surrounding viscous fluid. For each swimmer, we measured several emergent kinematic paramaters, including tail beat amplitude, body wavelength, body wave speed, and the neuromechanical phase lag, according to previous methods [33]. Each parameter was calculated during tail beats in which the swimmer was moving steadily (after 6 tail beats). Tail beat amplitude, $A$, was measured by taking the distance between extreme lateral positions of the tail tip. Body wavelength and wave speed were measured by tracking the distance between opposite signed peaks in curvature. The neuromechanical phase lag is defined as $1-V_{\text {mech }} / V_{a c t}$, where $V_{\text {mech }}$ is the mechanical body wave speed (measured based on the speed of the curvature wave along the body) and $V_{\text {act }}$ is the neural activation wave speed (set as a parameter in the simulations).

The Reynolds number (ratio of inertial to viscous forces in the system) Re was calculated according to $R e=U L / \nu$ where $U$ is the steady swimming speed of the lamprey, $L$ is the length of the lamprey 
body, and $\nu$ is the kinematic viscosity of the fluid. The Strouhal number (a dimensionless parameter characterizing vortex shedding) $S t$ was calculated according to $S t=f A / U$ where $f=1 \mathrm{~Hz}$ is the tail beat frequency, $A$ is the tail beat amplitude, and $U$ is the mean steady swimming speed.

\section{Results}

We examined the effects of four nonlinearities in muscle force production: the force-length and forcevelocity relationships [8,9, a work-dependent nonlinearity [12, and a calcium-depend change in passive stiffness 14,15 . For the first three relationships, we tested what happens when the nonlinearity is present or absent. For the stiffness of the series elastic element, we examine the effect when stiffness increases proportionally to the amount of calcium bound to the myosin filaments, or when stiffness is constant but low, or constant but high. Thus, there are twenty four combinations of the nonlinearities.

Simulations are coded with zeroes (0) or uppercase letters indicating which nonlinearities are included: " 0 " or " $V$ " for velocity dependence off or on, " 0 " or " $L$ " for length dependence off or on, " $O$ " or " $W$ " for work-dependent deactivation off or on, and " 0 ", " $S$ ", or " $\sigma$ " for low, high, or calcium-dependent passive stiffness (Figure 1). Figure6 summarizes the codes.

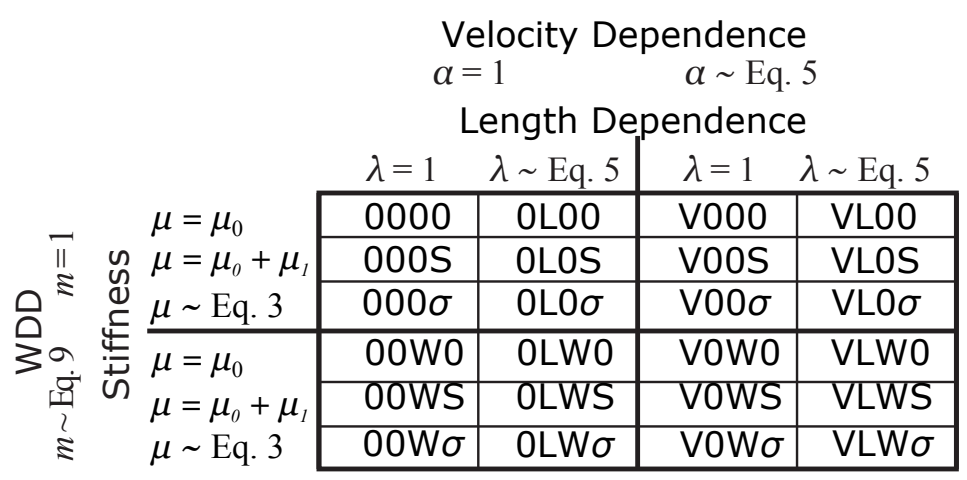

Figure 6. Simulation coding scheme. Each simulation is designated with four (4) characters indicating which nonlinear effects are present, where uppercase letters indicate the nonlinearity was present. See text for description.

\subsection{Nonlinearities reduce muscle force and stabilize oscillations}

To illustrate the effect of the nonlinearities, we show here a detailed comparison of two simulations: simulation $000 S$, in which all nonlinearities are off; and simulation $V L W \sigma$, in which all nonlinearities are 
on. Figure 7 shows the parameters involved in force development for each of the two simulations in a representative segment at the middle of the body.
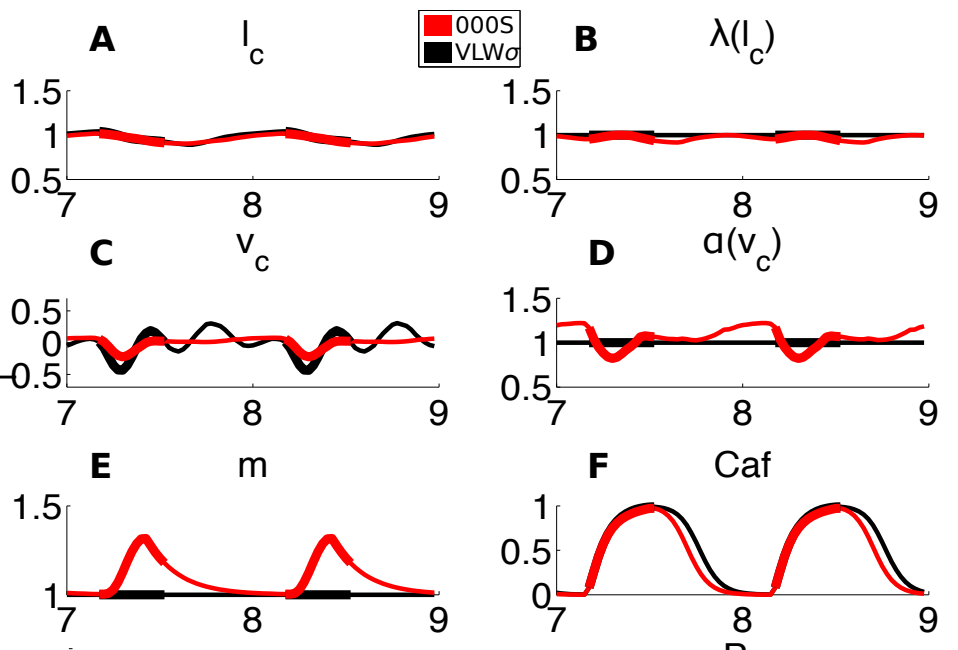

I
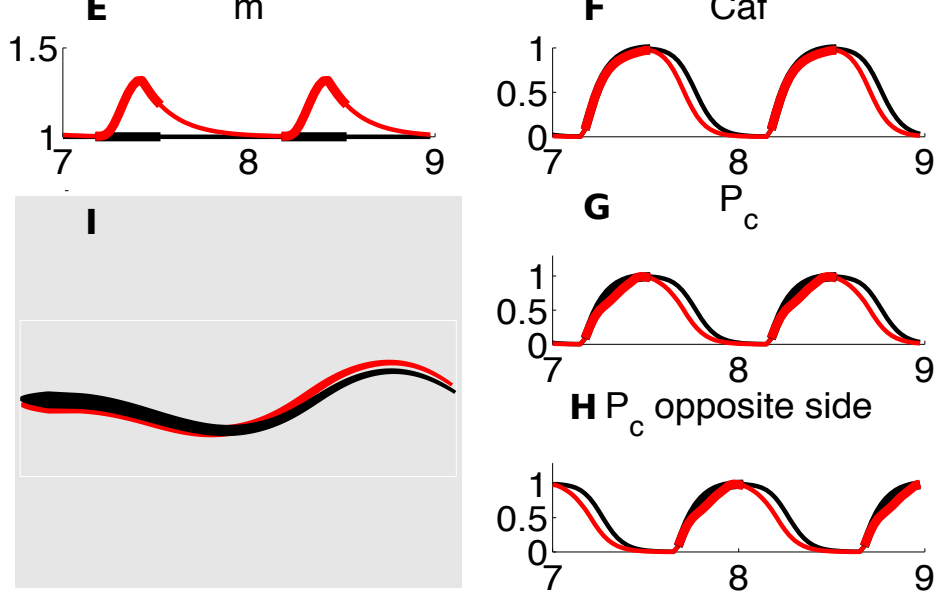

Figure 7. Comparison of swimmers with and without nonlinearities. Results for $t=7 \mathrm{~s}$ to $9 \mathrm{~s}$ averaged over the middle $18 \%$ of the body for simulations 000S (black; all nonlinearities off) and $V L W \sigma$ (red; all nonlinearities on). The thickened parts of the curves indicate the muscle is active. (A) Length of the contractile element, $l_{c}$, dimensionless having been normalized against the rest length of the segment. (B) Dimensionless length dependence factor $\lambda\left(l_{c}\right)$. (C) Velocity of the contractile element, $v_{c}$, with dimensions $s^{-1}$ having been normalized against the rest length of the segment. (D) Dimensionless velocity dependence factor $\alpha\left(v_{c}\right)$. (E) Integrated muscle work $m$. (F) Relative concentration of dimensionless calcium-bound factors $C a f$. (G) $P_{c}$ - Force developed which has been normalized against maximum isometric force $P_{0}$. $(\mathrm{H})$ Force developed on the opposite side of the body. (I) Representative waveforms of $000 S$ (black) and $V L W \sigma$ (red).

For swimmer $000 S$, which is closest to the one studied by Tytell et al. [7], force development is only dependent on the amount of bound calcium (Caf), which is governed by the activation wave and a scaling factor that decreases the force closer to the tail, both of which are pre-set. Thus, the time course of force development is identical everywhere along the body and is not affected by the length or velocity of the segment. 
For swimmer $V L W \sigma$, the nonlinearities largely reduce the muscle forces (Figure $7 \mathrm{G}$ ), but also prevent oscillations in the force development of each segment (compare the red and black curves in Figure 7C). Despite the slightly lower force in the nonlinear case $(V L W \sigma)$, the muscle segment cycles through nearly the same range of lengths, but it does so somewhat more slowly and smoothly than in o00S (Figure $7 \mathrm{~A}$ and C). In this case, length- and velocity-dependent nonlinearities are important (Figure 7B and D) in stabilizing force production. McMillen et al. 29, in a related lamprey model, observed a similar but much stronger effect: body oscillations grew unrealistically large when length- and velocity-dependent effects were removed.

Figure $7 \mathrm{E}$ and $\mathrm{F}$ show the effect of work-dependent deactivation: after the muscle does positive work, $m$ increases, which causes Caf, the muscle activation term, to decrease more rapidly. Together, workdependent deactivation and the length and velocity dependence reduce and stabilize the muscle force (Figure $7 \mathrm{G}$ ), particularly during the deactivation period.

Figure 8 shows the body motion and flow patterns developed by the two swimmers (see also supplemental movies S1 and S2). The basic movements of the body are similar, but the swimmer without nonlinearities $(000 S)$ swims very slightly faster (gray swimmer in Figure 8), primarily due to the higher forces $\left(0.51 \mathrm{~L} \cdot \mathrm{s}^{-1}\right.$ vs $\left.0.50 \mathrm{~L} \cdot \mathrm{s}^{-1}\right)$. The wakes are also structurally similar, with a strong primary vortex shed each time the tail changes direction and two smaller secondary vortices shed as the tail sweeps across. There are also differences during the acceleration period (about $0 \mathrm{~s}$ to $5 \mathrm{~s}$ ) so that the model with no nonlinearities accelerates more quickly. The two effects allow the lamprey without nonlinearities to pull ahead of the nonlinear model by $t=6.0 \mathrm{~s}$. However the cost of the swimming is reduced by the presence of the nonlinearities so that it will take much less work to swim the same absolute distance.

Calcium-dependent changes in stiffness have relatively little effect on the swimming kinematics or the energetics. Calcium rises rapidly during activation, so that the variable stiffness case is quite similar to the maximum stiffness case (compare cases with $\sigma$ to those with $S$ ).

\subsection{Velocity dependence reduces peak forces near the tail}

Figure 9 shows how the peak normalized force changes along the body for several simulations. Because velocities are higher near the tail and the length changes are greater, the length and velocity dependences tend to reduce the peak normalized force. Total force always declines near the tail because it is proportional to the cross-sectional area, which also decreases near the tail; the values shown in Figure 9 
represent the force scaled by the peak isometric force at that point along the body. The force decrease is most pronounced for swimmers with both length and velocity dependence ( $V L W \sigma$, red curve), but the decrease is also present for those with just velocity dependence ( $V 0 W \sigma$, blue curve; and $V 00 \sigma$, cyan curve). Work-dependent deactivation decreases the peak force, but its effect is fairly constant along the

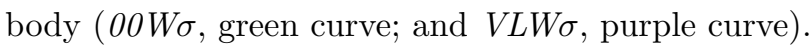

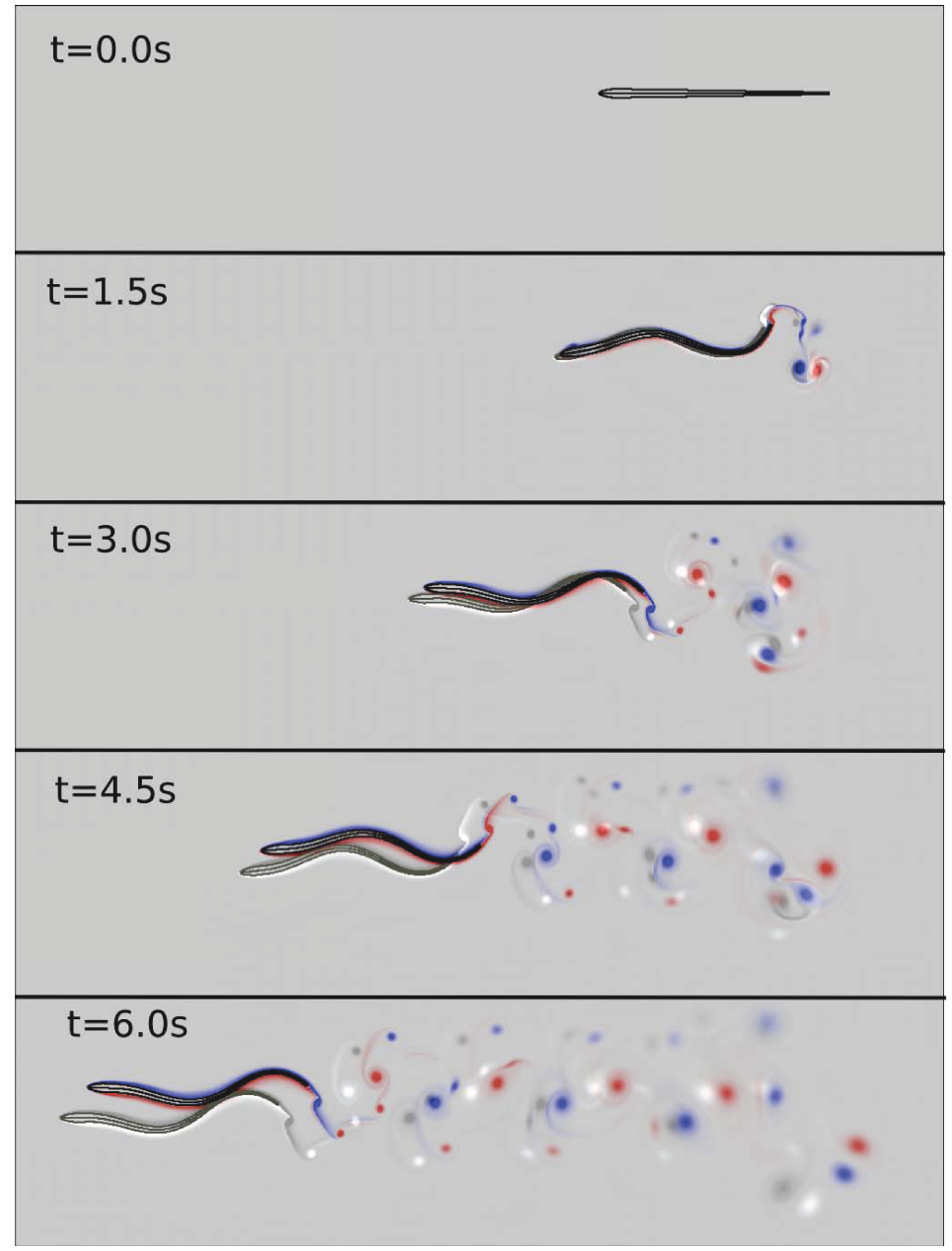

Figure 8. Snapshots of the flow patterns for two overlaid simulations with and without nonlinearities. Swimmer $000 S$, which has all nonlinearities off, is shown in gray with vorticity in dark gray (clockwise) and white (counterclockwise). Swimmer $V L W \sigma$ is shown in black with vorticity in blue (clockwise) and red (counterclockwise). See also swimming dynamics and vorticity wakes for 10 seconds of simulated time for 000S (Movie S1) and VLW $\sigma$ (Movie S2) in the Supporting Information. 


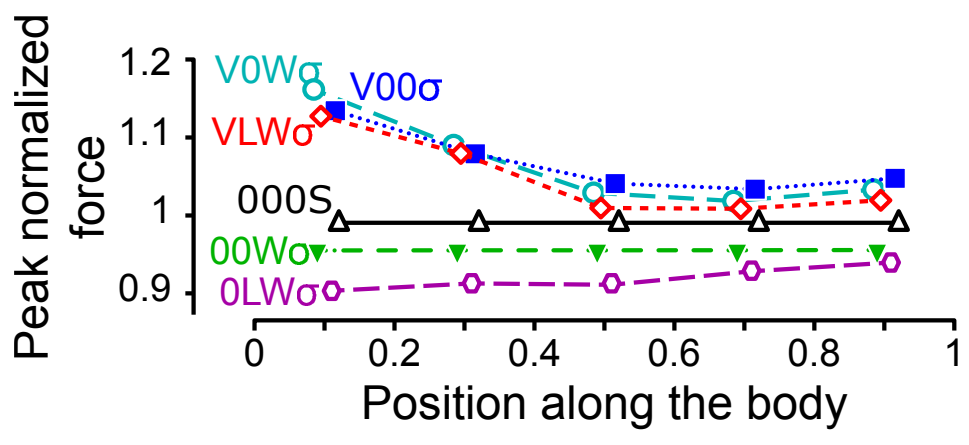

Figure 9. Velocity dependence causes peak normalized muscle force to decrease along the body. Normalized force (relative to peak isometric force) in six different swimmers, averaged over five regions along the body. Points are offset slightly so all of them can be seen.

\subsection{Velocity dependence decreases energy costs}

The nonlinear velocity dependence $(\alpha)$ has the largest effect of the four nonlinearities, because it reduces both the energy cost for swimming a unit distance and the metabolic power used to overcome drag. For each of the 24 different combinations of nonlinearities (Figure 6), we measured the kinematics and estimated the cost of transport (energy required to transport a unit mass over a unit distance: Equation 22 and the nondimensional power coefficient (the muscle power used relative to the power required to overcome drag: Equation 24), both accounting for the lower metabolic energy required for eccentric vs. concentric contractions 32 .

Simulations with velocity dependence $(\alpha)$ and work-dependent deactivation $(m)$ tended to have the lowest cost of transport (Figure 10). Power coefficients were also low for many of these swimmers, as long as the series elastic element was sufficiently stiff (simulations with $S$ or $\sigma$ ). Swimmers with soft springs (simulations with 0 at the end) generally swam slowly, and so their power coefficients were much higher.

\subsection{Work dependent deactivation reduces co-contraction}

In our model, muscles are always activated for $36 \%$ of the tail beat cycle. Muscle force, however, takes time to decay to zero after activation ceases. If the force on one side remains on for too long, it can end up resisting force development on the opposite side and increasing energy costs. Moreover, if the muscle produces force after the end of activation for too long, it can end up producing force as the muscle on the opposite side forcibly lengthens it (an effect called an "eccentric contraction"), resulting in an increase in force due to the velocity dependence. 


\begin{tabular}{|c|c|c|c|c|}
\hline $\mathbf{A}$ & 00 & $\mathrm{OL}$ & vo & $\mathrm{VL}$ \\
\hline 00 & 61 & 25 & 21 & 14 \\
\hline & 61 & 59 & 20 & 23 \\
\hline & 61 & 57 & 19 & 24 \\
\hline & 41 & 29 & 10 & 8 \\
\hline & 49 & 49 & 15 & 16 \\
\hline & 49 & 47 & 20 & 21 \\
\hline
\end{tabular}

\begin{tabular}{|c|c|c|c|c|c|}
\hline B & 00 & $\mathrm{OL}$ & V0 & VL & \\
\hline 00 & 1.26 & 2.60 & 1.16 & 7.51 & ighest \\
\hline OS & 1.26 & 1.26 & 0.41 & 0.48 & $10-50 \%$ \\
\hline $0 \sigma$ & 1.26 & 1.23 & 0.39 & 0.49 & $0-10 \%$ \\
\hline W & 1.10 & 1.65 & 0.66 & 1.28 & ]0-10\% \\
\hline w & 1.05 & 1.09 & 0.32 & 0.33 & 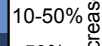 \\
\hline Wo & 1.06 & 1.05 & 0.41 & 0.44 & lowest \\
\hline
\end{tabular}

Figure 10. Both the cost of transport and the power coefficient are lowest in simulations with velocity dependence and work dependent deactivation (A) Figure of cost of transport values in $\mathrm{mJ} \cdot \mathrm{cm}^{-1} \cdot \mathrm{g}^{-1}$ during steady swimming. (B) Figure of power coefficients, muscle power relative to power required to overcome drag at the average swimming speed. All simulations are compared to the reference model (simulation 000S, shown with a dotted line around it), which is closest to the one studied in [7]. Darker blue colors represent a larger decrease relative to the reference, while darker red colors represent a larger increase.

If WDD is not present, these eccentric contractions can produce a substantial amount of force, which takes energy. If WDD is present, muscle on one side of the body turns off rapidly enough that the force from the other side does not overlap. This coupling between WDD and velocity dependence is present in most of the simulations, but it is most obvious when comparing simulations VLOO and VLWO (Figure 11). Of all the simulations, VL00 uses the most power relative to drag; adding WDD (simulation $V L W 0$ ) reduces the power almost 6 times. Figure $11 \mathrm{~B}$ shows that calcium on the left side stays bound to the myosin filaments much longer without WDD (VL00; black lines). When muscle on the right begins producing force, Caf is still sufficiently high on the left so that the left-side muscle produces force again through an eccentric contraction, resulting in an extra bump in the force curve (black curve, Figure $11 \mathrm{G}$ ). The extra force production is costly, both because it requires energy itself and because it reduces the overall tail beat amplitude.

\subsection{Effective swimming kinematics are similar, even when energy costs differ}

Most of the simulations had similar swimming kinematics, even when the energy costs were very different. As long as the series elastic element was stiff $(S)$ or varied in stiffness $(\sigma)$, swimmers generally reached a swimming speed of approximately $0.5 \mathrm{~L} \cdot \mathrm{s}^{-1}$, a tail beat amplitude of about $0.12 \mathrm{~L}, R e$ around 8000 , and a Strouhal number of around 0.5. Wave speed and wavelength were fairly consistent at $0.75 \mathrm{~L} / \mathrm{s}$ and $0.8 \mathrm{~L}$, resulting in a neuromechanical phase lag of about 0.14 . The swimmers with the much softer 


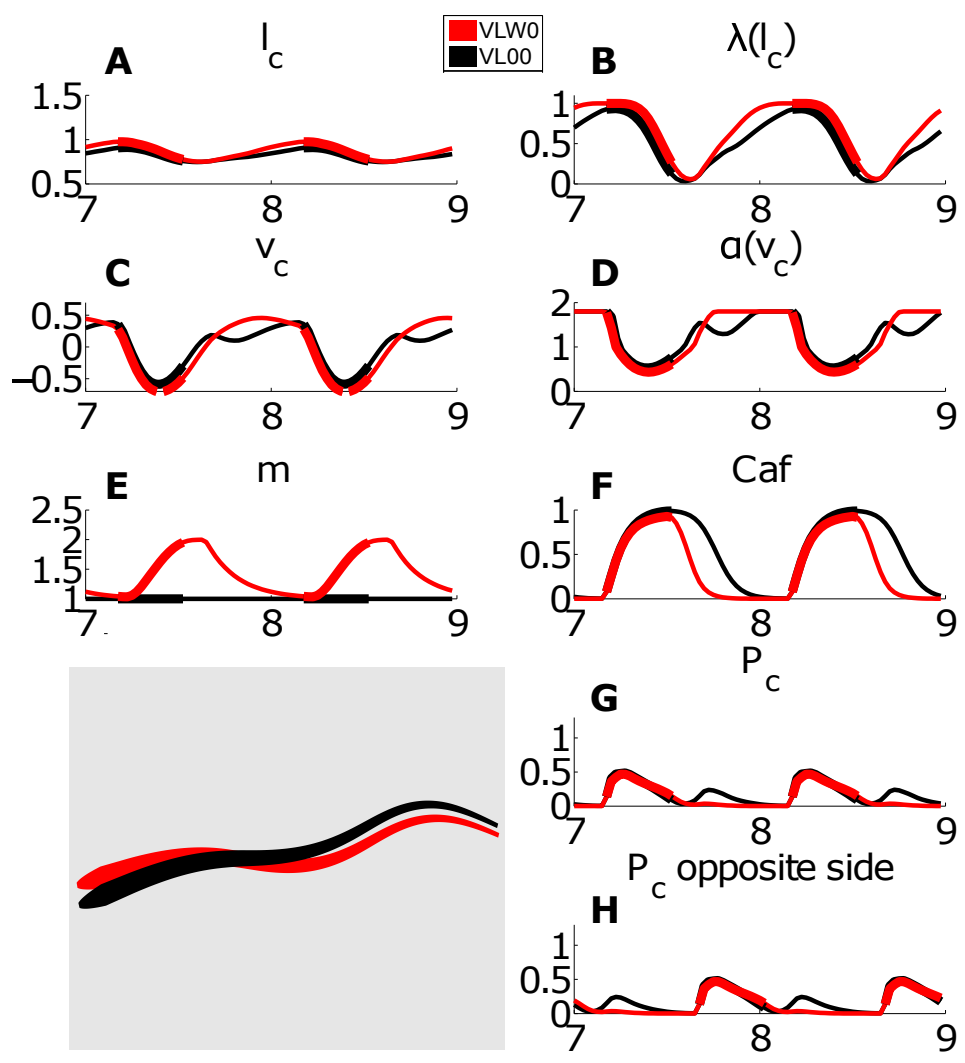

Figure 11. The effect of work dependent deactivation on co-contraction of muscles on opposite sides of the body. Results for $t=7 \mathrm{~s}$ to $9 \mathrm{~s}$, averaged over the middle $18 \%$ of the body, for simulations VLOO (black) and VLWO (red). The thickened parts of the curves indicate the muscle is active. (A) Length of the contractile element, $l_{c}$, dimensionless having been normalized against the rest length of the segment. (B) Dimensionless length dependence factor $\lambda\left(l_{c}\right)$. (C) Velocity of the contractile element, $v_{c}$, with dimensions $s^{-1}$ having been normalized against the rest length of the segment. (D) Dimensionless velocity dependence factor $\alpha\left(v_{c}\right)$. (E) Integrated muscle work $m$. (F) Relative concentration of dimensionless calcium-bound factors Caf. (G) $P_{c}$ - Force developed which has been normalized against maximum isometric force $P_{0}$. $(\mathrm{H})$ Force developed on the opposite side of the body. (I) Representative waveforms of $V L 00$ (black) and $V L W 0$ (red).

spring (simulations coded with 0 at the end), had much lower tail beat amplitudes $(0.08 \mathrm{~L}$ on average) and swimming speeds $\left(0.30 \mathrm{~L} \cdot \mathrm{s}^{-1}\right)$, corresponding to a much higher Strouhal number (0.60). Body wavelength in these swimmers was shorter $(0.67 \mathrm{~L})$, leading to a larger neuromechanical phase lag $(0.281)$.

Despite the similar movements, some swimmers used dramatically more muscular energy to achieve these kinematics. For example, swimmers without velocity dependence tended to use 2 to 3 times more energy than those with velocity dependence (Figure 10p. 


\section{Discussion}

We studied the effects of four different muscle nonlinearities (Figure 1) on the cost of transport and kinematics of a computational swimmer, modeled after the lamprey. We examined the roles of the length and velocity dependence of muscle force production 8; a history dependence, in which muscles deactivate more rapidly after they have produced positive mechanical work 12 ; and a newly described calcium dependence in the stiffness of the passive elastic element 14 15]. Cost of transport is highest when none of the nonlinearities are present, and can decrease by almost six times when all of the nonlinearities are included (Figure 10p.

All of the muscle nonlinearities reduced peak forces. Even the velocity dependence, which can potentially increase force during eccentric (lengthening) activity, does not increase the peak, because the peak force occurs during shortening. Indeed, all of the swimmers with nonlinearities swam at the same speed or slower than the one with all of the nonlinearities off $(000 S)$. In a mathematical model of hopping driven by a similar Hill-type muscle model, Haeufle et al. 19 made a similar observation: intrinsic muscle nonlinearities reduced the hopping height.

\subsection{The velocity dependence and work dependent deactivation work together to cause the largest decrease in energy cost}

The reduction in forces leads to a corresponding reduction in energy production, and the velocity dependence causes the largest part of the reduction. When muscle force does not depend on velocity, the muscles tend to produce high forces, even when they are contracting rapidly, which requires more power. They also cannot produce higher forces during active lengthening. When muscle force does depend on velocity, the power drops off at high speed and muscles can efficiently resist lengthening. Both of these effects combine to reduce the overall cost of transport in simulations with velocity dependence. The velocity dependence also seems to stabilize muscle length changes, as swimmers without velocity dependence exhibit more oscillations in the length or velocity (see Figure 7).

Surprisingly, swimmers with very similar kinematics can have different energetic requirements. Most swimmers with relatively stiff series elastic elements have similar kinematics. However, those with the velocity dependence achieve nearly identical kinematics while using about half the metabolic energy as those without velocity dependence (Figure 10). Although velocity dependence is a universal feature of 
muscle, if different animals have different shapes to their force-velocity relationships, or if those shapes change with age, exercise or temperature, that may dramatically affect the cost of transport even if the swimming movements are similar. Here we chose to focus on a force-velocity relationship based on fitted data [9]. Because velocity dependence has a marked effect on the energetics of swimming in this model,

the effects of the functional form of the force-velocity relationship on energetics will be explored in future work.

Muscle deactivation is also crucial. If muscles deactivate too slowly, the end of force development on one side of the body can overlap with the beginning of force development on the opposite side. In extreme cases (Figure 11), this can lead to a second peak in force, as seen in VL00 but not VLW0, due to the velocity dependence. When these eccentric contractions are caused by antagonists fighting one another, they increase energy consumption.

\subsection{The neuromechanical phase lag does not cause the energy decrease}

However, in our previous work [3.7, we found that eccentric contractions could reduce energy consumption, when they do not oppose other muscles, but instead oppose fluid dynamic forces. Specifically, when muscle forces are relatively low compared to fluid forces, muscle near the tail ends up being active during lengthening; this phase lag between activation and body motion correlates with low cost of transport 3$]$. Similar simulations from Williams and McMillen 34, using the same muscle model, but a simple fluid model, found the same effect: as muscle forces decrease, the phase lag increases. In the current work however, energy savings do not come from this neuromechanical phase lag. Indeed, nearly all of the faster swimmers have approximately the same phase lag. Instead, energy savings in the current work largely comes from decreases in co-contraction.

In the current model, we did not change the overall body stiffness of the swimmer or the peak muscle forces, but we did alter the stiffness of the series elastic element. If this element is very soft, the swimmer is not able to move. Our tests suggest the stiffness of the series elastic element does not greatly affect performance as long as the maximum stiffness is not too low. If the elastic element stiffness is too low, then most of the contraction energy is lost and force development is greatly reduced. We suspect that the effects of variable stiffness may be more important for the response to perturbations or for unsteady motions. Further studies will be necessary to clarify these effects. 


\subsection{Comparison to previous models}

McMillen et al. [29] examined the effects of length and velocity dependence on a swimming lamprey driven by an earlier version of the current muscle model [10], but with a much simpler, resistive fluid dynamic model (after [35]). In the current study, the organism model is fully coupled to an incompressible, viscous fluid model in an immersed boundary framework. It has been shown that the force interaction between the structure and the fluid can be very different when the full fluid equations are considered rather than the simplified resistive force model [36]. When they removed the nonlinearities, it was also necessary to reduce the muscle force density to prevent the amplitude from increasing dramatically. Adding nonlinearities back in reduced the forces, particularly near the head, and decreased the swimming speed [29]. We also observed that the nonlinearities decreased muscle force, but the effect on amplitude was negligible, as long as the series elastic element was sufficiently stiff. Differently, we found that nonlinearities caused peak forces to decrease near the tail, not near the head (Figure 9.

Previous work has examined how muscle nonlinearities affect stability, and most find the force-velocity relationship enhances stability by providing a nonlinear damping effect (e.g., 6, 17 19, 37]). Antagonistic muscles also contribute to stability, provided they have typical force-velocity and force-length relationships [38. We also find the force-velocity relationship damps out extra oscillations in our swimmer (see Figure 7].

To our knowledge, no other study has directly examined how intrinsic muscle nonlinearities contribute to mechanical energy production and consumption during locomotion. Woledge [20] suggested a steeper force-velocity curve could contribute to more effective conversion of ATP to force. We also find the forcevelocity curve contributes to efficient swimming, though we do not directly include metabolic energy. Many studies have shown running animals can benefit from elastic energy storage in some of the tendons and muscles in their lower legs (e.g., 39,40). Such leg muscles tend to contract isometrically, using relatively little metabolic energy to produce force [40], and allowing tendons to stretch and rebound during the running cycle. Such effects are not well understood for fishes or other swimming animals [41], though they may be present in some species like dolphins [42]. Our simulations are somewhat different. We suggest the intrinsic muscle nonlinearities, particularly the force-velocity relationship, contribute to energy economy by reducing the power consumed at high shortening speeds and increasing the force during active lengthening, which requires relatively little metabolic energy. 


\subsection{The determinants of muscle energy consumption}

In an influential review, Josephson 43 identified the force-length and force-velocity effects, along with activation, as the primary determinants of muscle power, and suggested that work-dependent deactivation was a secondary effect. Because we examined energy, our results do not directly bear on the determinants of power, which is the rate of energy production. However, for muscle energy production, our results suggest that force-velocity dependence may be a primary determinant, while work-dependent deactivation has an important secondary role. The force-length effect may be a primary determinant, but because the muscle does not change length substantially, its effect is limited. The length changes should be close to those in actual fishes, because the kinematics are very similar, and previous studies have shown that kinematics are a good predictor of muscle length changes [44]. The time course of activation is also likely to be a primary determinant, as Josephson argued [43, but we did not vary it. However, our results suggest that the timing of activation, or more precisely the timing of deactivation, is very important for reducing energy consumption. When opposing muscles deactivate slowly, they end up fighting each other and increasing energy consumption. Work-dependent deactivation causes muscles to deactivate rapidly and fight each other less (Fig. 11), but an actual animal could shorten the activation duration, which would have a similar effect.

Finally, Josephson pointed out the crucial importance of the coupling to load 43. Muscles do not operate in isometric, isovelocity, or even work loop conditions, but rather, their shortening velocity is determined by the inertial dynamics of other muscles, the body, and the environment. As discussed above, the coupling to load can reduce energy consumption. When muscle forces are relatively low compared to fluid forces, the neuromechanical phase lag is high [3,34 and the cost of transport is low. Recent work by Clemente and Richards [45] has shown that this coupling, particularly in a fluid environment, can limit the maximum muscle power. Our fluid model is based on the full Navier-Stokes equations, not a reduced model, and therefore should accurately represent the dynamic load on the muscle. However, our model is two dimensional, and represents a slice through the horizontal midline of a fish with roughly constant dorso-ventral cross-section. This simplification approximates eels and lampreys well [7, 46], but cannot account for more complex body shapes of other fishes. The size and shape of the body, peduncle, and tail fin will definitely affect the dynamic loading of the muscle and thus the energetics. These effects will be explored in a future three-dimensional model. 


\subsection{Conclusions and future directions}

Our results suggest that the force-velocity relationship, and particularly the force produced during lengthening, is a crucial aspect of energy economy for swimming animals, and probably also for legged animals. In an eccentric contraction, a muscle opposes some external force that lengthens it. The external force can either come from an antagonistic muscle, in which case it increases the energy cost of locomotion, or the force can come from interaction with the environment. In the second case, if the eccentric contraction is timed correctly, our previous results 3,7 suggest that it decreases the energy cost. We found that the work-dependent deactivation and velocity-dependence both decreased the opportunity for co-contraction, which can divert energy to overcoming resistance to force development on the shortening side. Since the properties of muscles and the non-linear dependencies are similar across most animals, these effects may be generally important for all animal locomotion.

Finally, the current model has a fixed activation pattern and does not take into account the effects of sensory feedback on muscle activity, and ultimately on the kinematics and energetics of swimming. It is well known that animals use proprioceptive sensory feedback to alter neural activation signals that control force development in muscles. These effects have been studied in reduced preparations or by severing afferent nerves for a long time (e.g., 47 [50]), but only recently have we started to develop the computational and experimental tools to study them in normal closed-loop behavior (see reviews in [4,51]). We expect incorporating proprioceptive information in the form of a more detailed neural activation model will change the energetics the swimming lamprey model by altering the relative amounts co-contraction and eccentric contractions opposing environmental forces. These effects will be explored in future work.

\section{Acknowledgments}

The authors would like to thank Avis Cohen and Thelma Williams for many helpful discussions, Boyce Griffith for his assistance with IBAMR, and Chia-Yu Hsu for her assistance in navigating the original lamprey model. 


\section{References}

1. Dickinson MH, Farley CT, Full RJ, Koehl MA, Kram R, et al. (2000) How animals move: an integrative view. Science 288: 100-6.

2. Tytell ED, Holmes P, Cohen AH (2011) Spikes alone do not behavior make: Why neuroscience needs biomechanics. Curr Opin Neurobiol 21: 816-822.

3. Miller LA, Goldman DI, Hedrick TL, Tytell ED, Wang ZJ, et al. (2012) Using computational and mechanical models to study animal locomotion. Integ Compar Biol 52: 553-575.

4. Cowan NJ, Ankarali MM, Dyhr JP, Madhav MS, Roth E, et al. (2014) Feedback control as a framework for understanding tradeoffs in biology. Integ Compar Biol : icu050.

5. McMahon TA (1984) Muscles, Reflexs, and Locomotion. Princeton University Press.

6. Brown I, Loeb G (2000) A reductionist approach to creating and using neuromusculoskeletal models. In: Winters J, Crago P, editors, Biomechanics and Neuro-Control of Posture and Movement, New York: Springer-Verlag. pp. 148-163.

7. Tytell ED, Hsu C, Williams TL, Cohen AH, Fauci LJ (2010) Interations between internal forces, body stiffness, and fluid environment in a neuromechanical model of lamprey swimming. Proc Nat Acad Sci USA 107: 19832-19837.

8. Hill AV (1938) The heat of shortening and the dynamic constants of muscle. Proc Roy Soc Lond B 126: 136-195.

9. Williams TL (2010) A new model for force generation by skeletal muscle, incorporating workdependent deactivation. J Exp Biol 213: 643-650.

10. Williams TL, Bowtell G, Curtin NA (1998) Predicting force generation by lamprey muscle during applied sinusoidal movement using a simple dynamic model. J Exp Biol 201: 869-875.

11. Chen J, Tian J, Iwasaki T, Friesen WO (2011) Mechanisms underlying rhythmic locomotion: dynamics of muscle activation. J Exp Biol 214: 1955-1964. 
12. Josephson RK, Stokes DR (1999) Work-dependent deactivation of a crustacean muscle. J Exp Biol 202: $2551-2565$.

13. Yeo SH, Monroy JA, Lappin AK, Nishikawa KC (2013) Phenomenological models of the dynamics of muscle during isotonic shortening. J Biomech 46: 2419-2425.

14. Monroy JA, Powers KL, Gilmore LA, Uyeno TA, Lindstedt SL, et al. (2012) What is the role of titin in active muscles? Exercise Sport Sci Rev 40: 73-78.

15. Nishikawa KC, Monroy JA, Uyeno TE, Yeo SH, Pai DK, et al. (2012) Is titin a 'winding filament'? A new twist on muscle contraction. Proc Roy Soc Lond B 279: 981-990.

16. Herzog W, Kamal S, Clarke HD (1992) Myofilament lengths of cat skeletal muscle: Theoretical considerations and functional implications. J Biomech 25: 945-948.

17. Wagner H, Blickhan R (1999) Stabilizing function of skeletal muscles: an analytical investigation. J Theor Biol 199: 163-179.

18. Chen L, Ren L (2010) The influence of intrinsic muscle properties on musculoskeletal system stability: A modelling study. J Bionic Eng 7: S158-S165.

19. Haeufle D, Grimmer S, Seyfarth A (2010) The role of intrinsic muscle properties for stable hopping - stability is achieved by the force-velocity relation. Bioinsp Biomimet 5: 016004(1-11).

20. Woledge RC (1968) The energetics of tortoise muscle. J Physiol 197: 685-707.

21. Johnston IA, Sidell BD, Driedzic WR (1985) Force-velocity characteristics and metabolism of carp muscle fibres following temperature acclimation. J Exp Biol 119: 239-249.

22. Johnston IA, Salamonski J (1984) Power output and force-velocity relationship of red and white muscle fibres from the pacific blue marlin (Makaira nigricans). J Exp Biol 111: 171-177.

23. Holt NC, Azizi E (2014) What drives activation-dependent shifts in the force-length curve? Biol Lett 10: 20140651:1-4. 
24. Brughelli M, Cronin J (2007) Altering the length-tension relationship with eccentric exercise. Sports Med 37: 807-826.

25. Raj IS, Bird SR, Shield AJ (2010) Aging and the force-velocity relationship of muscles. Exp Gerontol 45: 81-90.

26. Gao F, Zhang LQ (2008) Altered contractile properties of the gastrocnemius muscle poststroke. J Appl Physiol 105: 1802-1808.

27. Kawamura Y, Ohtani Y, Maruyama K (1994) Biodiversity of the localization of the epitopes to connectin antibodies in the sarcomeres of lamprey, electric ray, and horse mackerel skeletal muscles. Tiss Cell 26: 677-685.

28. He J, Levine WS, Loeb GE (1991) Feedback gains for correcting small perturbations to standing posture. IEEE Trans Automat Contr 36: 322-332.

29. McMillen T, Williams T, Holmes P (2008) Nonlinear muscles, passive viscoelasticity and body taper conspire to create neuromechanical phase lags in anguilliform swimmers. PLoS Comput Biol 4: e1000157(1-16).

30. Peskin CS (2002) The immersed boundary method. Acta Numer 11: 479-517.

31. Griffith BE, Hornung RD, McQueen DM, Peskin CS (2007) An adaptive, formally second order accurate version of the immersed boundary method. J Comput Phys 223: 10-49.

32. Ruina A, Bertram JEA, Srinivasan M (2005) A collisional model of the energetic cost of support work qualitatively explains leg sequencing in walking and galloping, pseudo-elastic leg behavior in running and the walk-to-run transition. J Theor Biol 237: 170-192.

33. Tytell ED (2004) The hydrodynamics of eel swimming. II. Effect of swimming speed. J Exp Biol 207: $3265-3279$.

34. Williams TL, McMillen T (2015) Strategies for swimming: Explorations of the behaviour of a neuro-musculo-mechanical model of the lamprey. Biol Open 4: BIO20149621-258.

35. Taylor GI (1952) Analysis of the swimming of long and narrow animals. Proc R Soc Lond A 214: 158-183. 
36. Tytell ED, Borazjani I, Sotiropoulos F, Baker TV, Anderson EJ, et al. (2010) Disentangling the functional roles of morphology and motion in the swimming of fish. Integ Compar Biol 50: 11401154.

37. Blickhan R, Seyfarth A, Geyer H, Grimmer S, Wagner H, et al. (2007) Intelligence by mechanics. Phil Trans Roy Soc Lond A 365: 199-220.

38. Wagner H, Blickhan R (2003) Stabilizing function of antagonistic neuromusculoskeletal systems: an analytical investigation. Biol Cybernet 89: 71-79.

39. Alexander RM (1988) Elastic Mechanisms in Animal Movement. Cambridge University Press, 1 edition.

40. Biewener AA, Daley MA (2007) Unsteady locomotion: integrating muscle function with whole body dynamics and neuromuscular control. J Exp Biol 210: 2949-2960.

41. Tytell ED, Hsu CY, Fauci LJ (2014) The role of mechanical resonance in the neural control of swimming in fishes. Zoology 117: 48-56.

42. Pabst DA (1996) Springs in swimming animals. Amer Zool 36: 723-735.

43. Josephson RK (1999) Dissecting muscle power output. J Exp Biol 202: 3369-3375.

44. Coughlin DJ, Valdes L, Rome LC (1996) Muscle length changes during swimming in scup: Sonomicrometry verifies the anatomical high-speed cine technique. J Exp Biol 199: 459-463.

45. Clemente CJ, Richards C (2013) Muscle function and hydrodynamics limit power and speed in swimming frogs. Nature Comm 4: 1-8.

46. Gazzola M, Argentina M, Mahadevan L (2014) Scaling macroscopic aquatic locomotion. Nature Physics 10: 758-761.

47. Liddell E, Sherrington C (1924) Reflexes in response to stretch (Myotatic reflexes). Proc Roy Soc Lond B 96: 212-242.

48. Rossignol S, Dubuc RJ, Gossard JP (2006) Dynamic sensorimotor interactions in locomotion. Physiol Rev 86: 89-154. 
49. Prochazka A, Gillard D, Bennett DJ (1997) Positive force feedback control of muscles. J Neurophysiol 77: 3226-3236.

50. Grillner S, McClellan A, Perret C (1981) Entrainment of the spinal pattern generators for swimming by mechanosensitive elements in the lamprey spinal cord in vitro. Brain Res 217: 380-386.

51. Roth E, Sponberg S, Cowan NJ (2014) A comparative approach to closed-loop computation. Curr Op Neurobiol 25: 54-62. 